\title{
A network-based analysis for mining the risk pathways in glioblastoma
}

\author{
JING LI $^{1}$, YUJIE XIE ${ }^{2}$, CHI ZHANG ${ }^{2}$, JIANXIONG WANG $^{2}$, YONG WU $^{3}$, \\ YUAN YANG ${ }^{3}$, YANG XIE ${ }^{3}$ and ZHIYU LV ${ }^{3}$ \\ Departments of ${ }^{1}$ Hepatobiliary Surgery, ${ }^{2}$ Rehabilitation Medicine and ${ }^{3}$ Neurology, \\ Affiliated Hospital of Southwest Medical University, Luzhou, Sichuan 646000, P.R. China
}

Received November 1, 2018; Accepted June 13, 2019

DOI: $10.3892 / \mathrm{ol} .2019 .10598$

\begin{abstract}
The most malignant type of brain tumour is glioblastoma multiforme (GBM). Patients with GBM often have a poor prognosis, as a result of incomplete or inaccurate diagnoses. Regulatory pathways have been demonstrated to serve important roles in complex human diseases. Therefore, deciphering these risk pathways may shed light on the molecular mechanisms underlying GBM progression. In the present study, differentially expressed genes and microRNAs (miRNAs) in a publicly available database were identified between normal and tumour samples. To determine the pathophysiology and molecular mechanisms underlying GBM, integrated network analysis was performed to mine GBM-specific risk pathways. Specifically, a GBM-specific regulatory network was constructed that integrated manually curated GBM-associated transcription and post-transcriptional data resources, including transcription factors and miRNAs. A total of 1,827 differentially expressed genes and 30 miRNAs were identified. The differentially expressed genes were significantly enriched in a number of immune response-associated functions. Based on the GBM-specific regulatory network, 15 risk regulatory pathways containing not only known regulators, but also potential novel targets that might be involved in tumourigenesis were identified. Network analysis provides a strategy for leveraging genomic data to identify potential oncogenic pathways and molecular targets for GBM.
\end{abstract}

\section{Introduction}

As a biologically aggressive subtype of malignant glioma, glioblastoma multiforme (GBM) is the most common and lethal brain tumour in adults (1). GBM diffusely infiltrates the brain at an early time point, and its complexity and distinct

Correspondence to: Professor Zhiyu Lv, Department of Neurology, Affiliated Hospital of Southwest Medical University, 25 Taiping Street 25, Luzhou, Sichuan 646000, P.R. China

E-mail: sanyangkaitai24@126.com

Key words: network, risk pathways, glioblastoma, bioinformatics pathophysiology are facilitated and dictated by the unique brain milieu and cellular interactions $(2,3)$. In total, $>80 \%$ of GBM cases are primary tumours, which typically respond poorly to current therapeutic approaches (4). Although previous studies detected a number of events associated with initiation or progression $(5,6)$, identification of the key molecular targets underlying the regulatory mechanisms of GBM remains to be elucidated.

At present, the wealth of molecular information generated by massively parallel sequencing technologies provides a great opportunity for discovering novel biomarkers in different types of cancer $(7,8)$. Transcriptome analysis involving protein-coding genes and noncoding microRNAs (miRNAs/miRs) has proven to be a valuable first step for studying the genetic characteristics of various complex human diseases, especially in malignant gliomas $(9,10)$. For example, Lai et al (11) found that miRNA-210 could serve as a potentially non-invasive biomarker for the diagnosis and prognosis of GBM. An increased expression level of the protein-coding gene PLAUR was determined to be a valuable predictor of the mesenchymal GBM subtype (12). However, it is unlikely that a single dysfunctional gene would lead to abnormal phenotypes. Multiple aberrant gene-gene interactions may have co-operative effects in the development and progression of complex diseases such as cancer $(13,14)$.

Understanding the molecular events associated with activation of the risk pathways in patients with GBM may facilitate the development and clinical testing of potential therapeutic targets.

In the present study, differentially expressed genes and miRNAs were identified by analysing a large protein-coding gene and miRNA expression dataset comprised of normal and tumour tissues from GBM samples obtained from The Cancer Genome Atlas (TCGA). A GBM-specific regulatory network involving transcription factors (TFs) and miRNAs was built. The GBM-specific regulatory network showed a scale-free network with a small-world property. The key molecules in the regulatory network were significantly associated with immune-related functions. Linear risk pathways of GBM were identified through systems-level analyses of the regulatory network. The identified molecular risk pathways may be a potential resource for understanding the pathogenesis and aetiology underlying GBM. 


\section{Materials and methods}

Acquiring known GBM-related genes. The GeneCards database provides a comprehensive map of manually curated human disease genes (15). In total, 32 GBM-associated genes were obtained by searching the GeneCards database according to all descriptions and aliases of GBM, including 'GBM', 'glioblastoma multiforme', 'malignant brain tumour', 'gliomatosis' and 'high-grade glioma'. In addition, 41 experimentally validated GBM-related miRNAs from the miR2Disease database were retrieved (16), a manually curated database of miRNA deregulation in various human diseases.

Identification of differentially expressed genes and miRNAs. The mRNA and miRNA expression profiles for 378 patients with GBM were downloaded from TCGA database (https://portal.gdc.cancer.gov/). The dataset contained 378 tumour samples and 10 patient-matched normal samples. The genes were mapped to the Entrez Gene IDs for mRNA expression data using biomaRt software version 2.40 .0 (17). In total, 11,273 genes and 470 miRNAs were included in the subsequent analysis. A Student's t-test was used to identify significantly differentially expressed genes and miRNAs from the GBM expression profiles. All P-values were adjusted using Bonferroni correction to account for multiple comparisons. Only genes and miRNAs with an adjusted $\mathrm{P}$-value $<0.01$ and a fold change $>1.2$ were considered statistically significant.

Extraction of the GBM-specific regulatory network. A TF and miRNA regulatory network was constructed by integrating the molecular interaction associations between genes and miRNAs from four databases [miRTarBase (18); The Transcription Factor Database (19); miRecords (20); and TarBase (21)]. To ensure the reliability of the relationships between the curated regulations, each individual interaction presented is supported by the literature.

Although differential expression analysis is often used to screen disease candidate genes, previous studies have shown that a number of disease-associated genes do not always exhibit differential expression patterns in microarray experiments $(14,22)$. Therefore, a candidate risk regulatory network was constructed by connecting all of the risk-associated differentially expressed nodes with their immediate neighbouring non-differentially expressed nodes.

Mining for GBM-specific risk regulatory linear pathways. Linear risk pathways potentially associated with GBM were obtained according to the following criteria: i) The beginning of a risk pathway was defined as a gene or miRNA with a 0 -indegree (it could not be regulated by other genes or miRNAs); and ii) the end of a risk pathway was defined as a gene or miRNA with a 0 -outdegree (it could not regulate other genes or miRNAs). In the GBM-specific regulatory network, all linear regulatory pathways were defined as pathways from the beginning node to the end node and were detected using $\mathrm{R}$ (23).

Functional enrichment analysis. The $\mathrm{R}$ package 'clusterProfiler' (24) was used to examine the significantly enriched gene ontology (GO) biological functions (25) of the differentially expressed genes. The adjusted P-values were calculated using the Benjamini and Hochberg method.

\section{Results}

Differential expression analysis of mRNAs and miRNAs. To detect potential key molecules underlying the pathology of GBM, the mRNA and miRNA gene profiles of patients with GBM from TCGA database were downloaded and differential expression analysis was performed. In total, 1,827 and 30 significantly differentially expressed genes and miRNAs were detected, respectively. The number of upregulated genes, $(1,085)$ was greater than the number of downregulated genes (742). Conversely, the number of upregulated miRNAs (11) was smaller than the number of downregulated miRNAs, 19 (Fig. 1). These results suggested that a number of candidate molecules may contribute to the carcinogenesis of GBM, and that miRNAs may serve a negative regulatory role.

Systematic characterization of a regulatory network specific to $G B M$. To examine the systems-level characteristics of the 'true' biological processes, including interactions and other combined activities associated with GBM, a comprehensive regulatory network among TFs, miRNAs and genes was built by combining four manually curated databases that focused on both transcriptional and post-transcriptional regulation. The background regulatory network was composed of 6,036 regulatory relationships. Of the background regulatory relationships, 1,827 differentially expressed genes and 30 differentially expressed miRNAs were considered as the risk seeds and mapped into the regulatory network to obtain a GBM-specific sub-network (Fig. 2). Given that networks provide knowledge that can be used to infer the information flow between regulators and target genes, a GBM-specific sub-network consisting of differentially expressed genes/miRNAs and their immediate neighbouring nodes from the TF-miRNA-gene regulatory network was further extracted. The GBM-specific regulatory network contained 160 interactions involving 23 TFs, 17 miRNAs and 105 target genes.

The network showed dense local interconnectivity and complied with a power-law distribution suggesting that the GBM-specific sub-network was scale-free, similar to most biological networks (26) (Fig. 3). The biological functions of the GBM-specific network were investigated. Functional enrichment analysis was performed, and the top 30 significantly enriched Gene Ontology functions are presented in Fig. 3. Notably, the results demonstrated a number of immune response-associated functions, including 'neutrophil-mediated immunity', 'neutrophil activation involved in immune response' and 'leukocyte differentiation'. Numerous studies have reported profound and generalised immunosuppression of GBM tumours, particularly within the context of cell-mediated immunity $(27,28)$. In addition, a number of other types of functions were found, such as 'axon development', 'haemostasis' and 'response to peptide', which might be unique or important to the brain. Together, these results support the possibility that the GBM-specific sub-network may be a potential resource for GBM-related research.

GBM-specific risk pathways. A biological interaction network contains a large amount of information, and its complex 


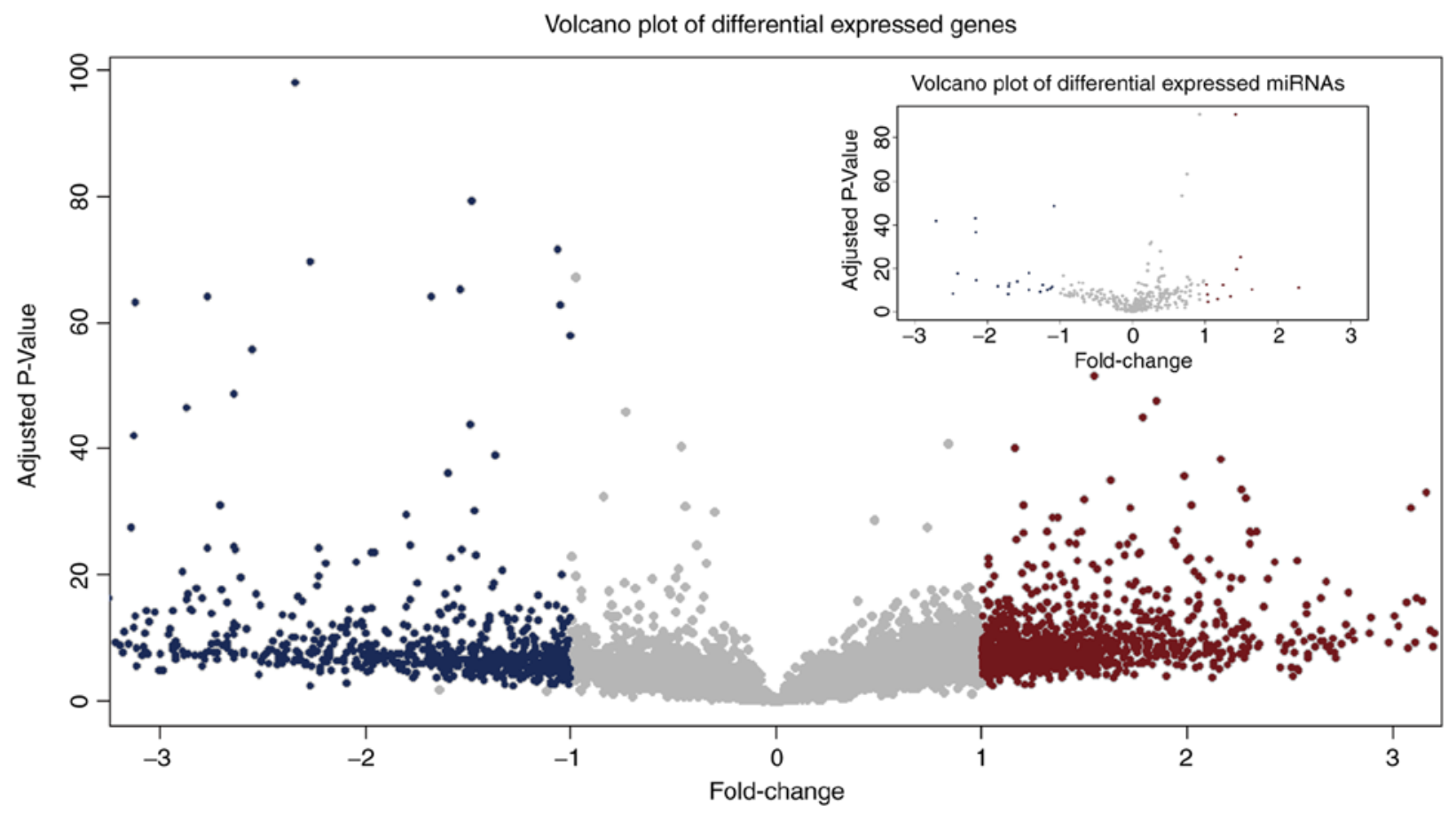

Figure 1. Volcano plot of the differentially expressed genes and miRNAs associated with glioblastoma multiforme. A total of 1,827 and 30 significantly differentially expressed genes and miRNAs, respectively were detected. Red dots represent genes and miRNAs with $\geq 1.2$-fold change increase and blue dots represent genes and miRNAs with $\leq 1.2$-fold decrease. miRNA, microRNA.

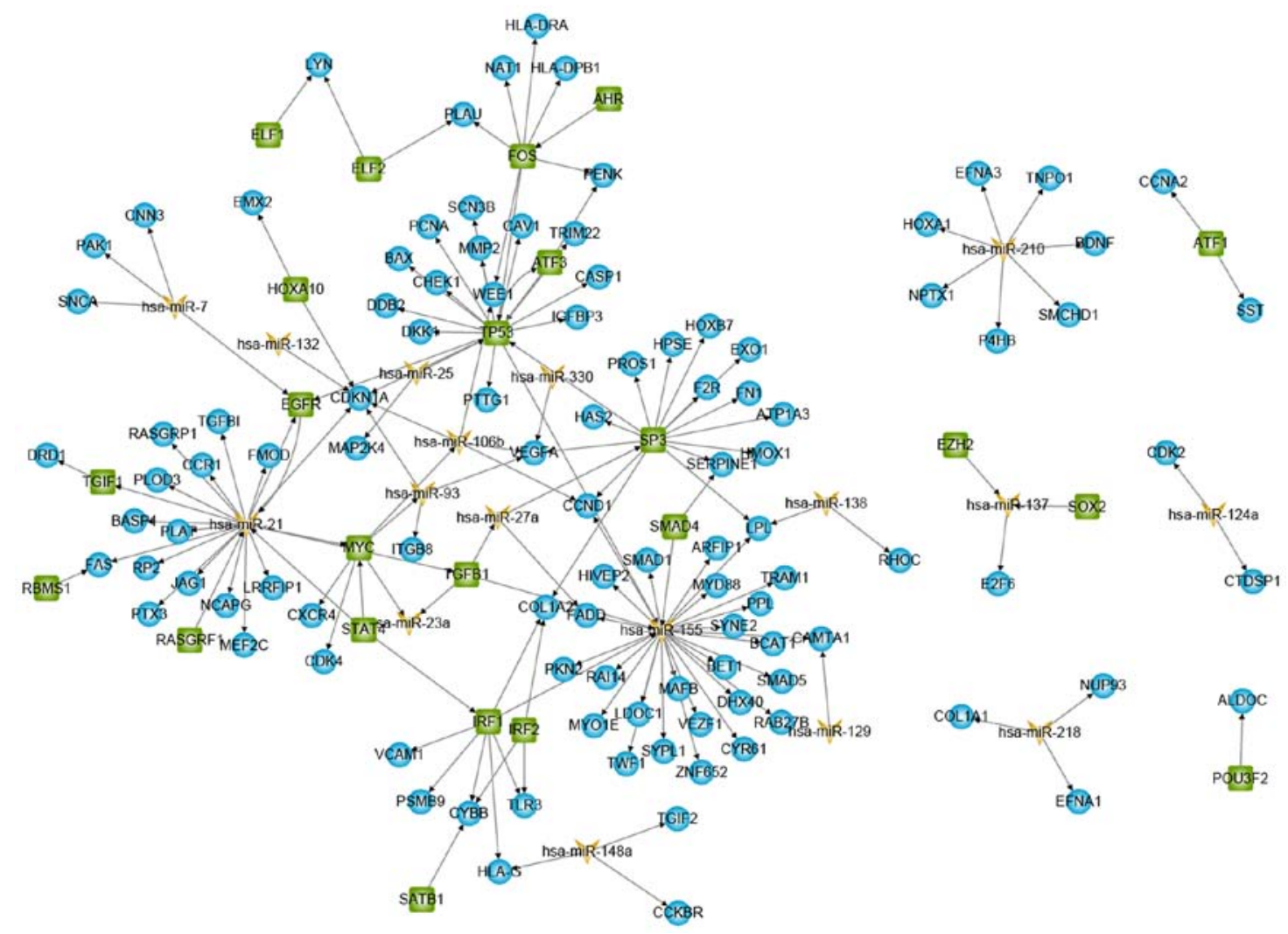

Figure 2. Glioblastoma multiforme-specific regulatory network. Green square nodes represent transcription factors, orange pointed nodes represent microRNAs and blue round nodes represent target genes.

structure may hinder the deduction of new insights for interested risk pathways. The risk pathways obtained from the regulatory network can shed light on the molecular mechanisms of GBM.
Detecting disease-associated pathways in a clear and simple manner is an important goal. In the present study, 615 linear risk pathways from the GBM-specific regulatory network 


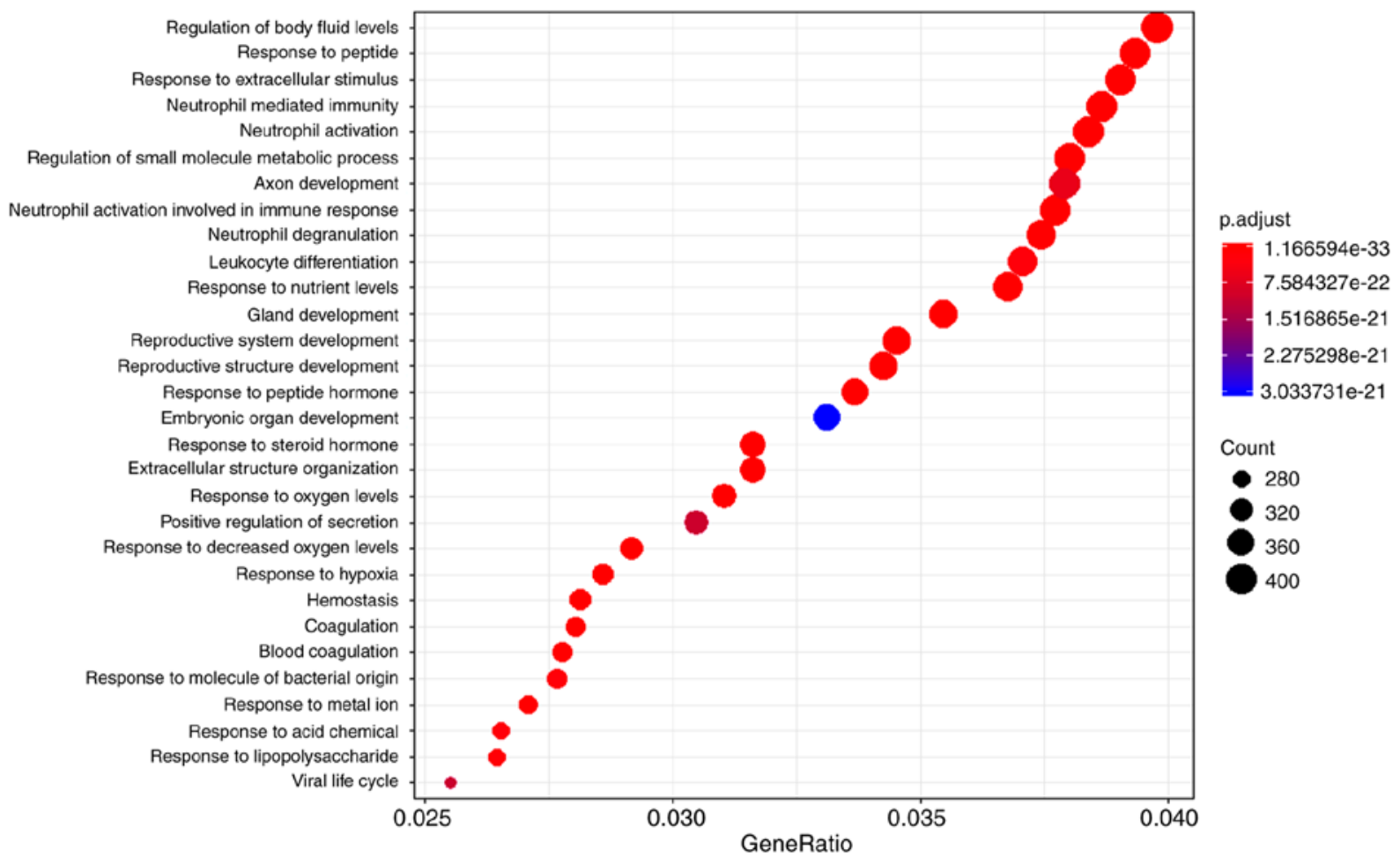

Figure 3. Characterization of the GBM-specific regulatory network. Top 30 significantly enriched biological processes in the GBM-specific regulatory network. The colour bar on the right represents the adjusted P-value and the circle size represents the number of genes.

were identified. Notably, a TF named EGFR was not only a known GBM disease gene, but also a differentially expressed gene (29). A total of 15-risk pathways were connected by EGFR and its up-stream regulator hsa-miR-7 (Fig. 4), which is a miRNA that has been reported as a prospective risk factor for $\operatorname{GBM}(30,31)$.

\section{Discussion}

Glioblastoma is an astrocyte-derived tumour with a propensity for malignancy and is caused by an abnormal disorder of multiple interacting genes rather than a single gene acting in isolation (32). Activation of molecular signalling cascades have been shown in GBM tumourigenesis (33). Identification of a cascade regulatory pathway, which usually has a clear and simple linear structure (34), may help reveal molecular mechanisms of the disease. A network-based approach to analyse risk regulatory pathways in GBM was used in the present study. Noncoding RNAs are emerging as key regulators of diverse biological processes and are important in cancer pathogenesis $(35,36)$. Therefore, noncoding RNAs involved in pathway regulation were also considered.

Through differential expression analysis, a total of 1,827 genes and 30 miRNAs were identified in normal and tumour tissues from GBM samples. When these candidate risk factors were taken as seed nodes, a GBM-specific regulatory network involving TFs, miRNAs and target genes was identified. Functional analysis showed that the GBM-specific network was significantly associated with several important functions of the immune response, axon development, haemostasis and response to peptides. From these, 15 reliable risk pathways specific to GBM were identified, which may be a useful

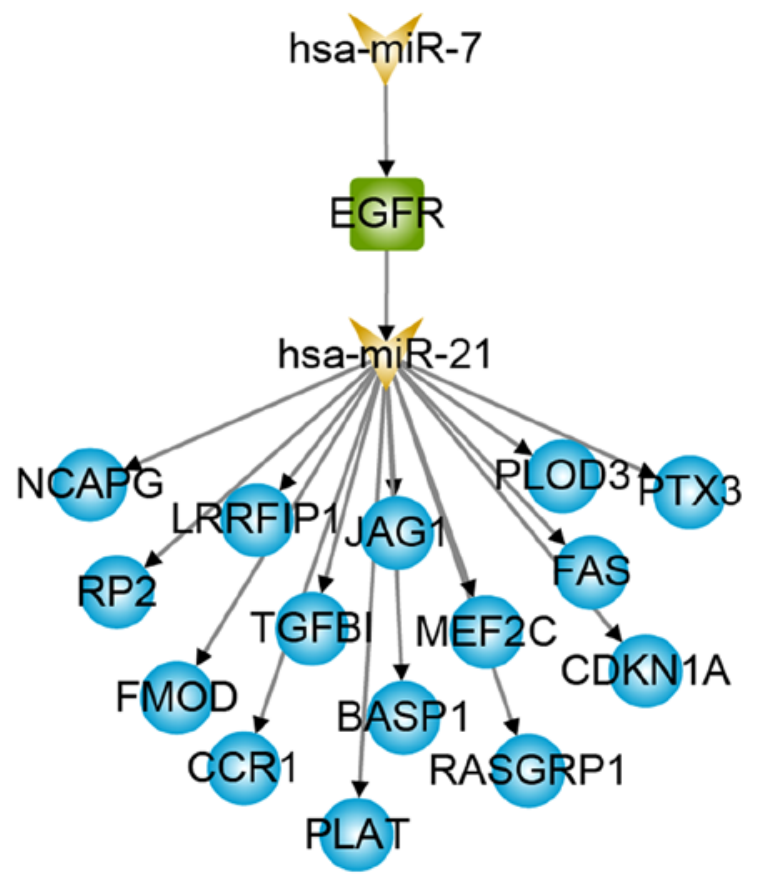

Figure 4. Risk regulatory pathways connected by EGFR and hsa-miR-7. EGFR, epidermal growth factor; miR, microRNA.

resource for increasing our fundamental understanding of the molecular role and regulation of GBM. These results showed that the 15 GBM-associated risk pathways were connected by a TF named EGFR that was both a known GBM gene and a differentially expressed gene. Numerous studies have highlighted the vital role played by EGFR during the emergence 
and development of GBM. Shao et al (37) showed that $\sim 80 \%$ of patients with GBM presented with EGFR genomic amplification or overexpression. Mischel et al (38) reported that EGFR-overexpressing GBMs promoted GBM cell proliferation, survival and angiogenesis. Wheeler et al (39) showed that EGFR inhibitors may be a novel therapeutic strategy for treating patients with GBM. In the present study, EGFR indirectly regulates 15 GBM-related genes (Fig. 4) and forms a cascade regulatory pathway with hsa-miR-7 and hsa-miR-21, participating in the occurrence and development of GBM. Regarding the downstream target genes, FAS-induced expression of chemokines in human glioma cells (40) and TGF $\beta 1$ gene expression are potential signatures for the mesenchymal high-grade glioma subtype (41). Therefore, the risk regulatory pathways miR-7, EGFR, miR-21 and FAS, and miR-7, EGFR, miR-21 and TGFB1 may cause disease by affecting the abnormal function of glioma cells.

In conclusion, accumulating evidence indicates that a single mutated or aberrantly expressed gene is not sufficient to result in an abnormal phenotype and that complex diseases are caused by the accumulative and co-operative effects of multiple dysregulated genes and gene interactions $(42,43)$. Risk regulatory pathways that consist of interacting genes with a linear chain structure can help reveal the molecular mechanisms of diseases. Through integration of transcriptional and post-transcriptional regulation, risk regulatory pathways in GBM that might serve as potential targets for clinical treatment were identified.

\section{Acknowledgements}

Not applicable.

\section{Funding}

No funding was received.

\section{Availability of data and materials}

The datasets used and/or analyzed during the present study are available from the author on reasonable request.

\section{Authors' contributions}

JL, YJX, YW, and YY collected and analyzed all data. CZ, YX and JXW performed statistical analysis. ZYL, JL and JXW contributed to the concept and design of the study and drafted the manuscript. JL, YW, and YY revised the manuscript. All authors read and approved the final manuscript.

\section{Ethics approval and consent to participate}

Not applicable.

\section{Patient consent for publication}

Not applicable.

\section{Competing interests}

The authors declare that they have no competing interests.

\section{References}

1. Parsons DW, Jones S, Zhang X, Lin JC, Leary RJ, Angenendt P, Mankoo P, Carter H, Siu IM, Gallia GL, et al: An integrated genomic analysis of human glioblastoma multiforme. Science 321: 1807-1812, 2008.

2. Choe G, Horvath S, Cloughesy TF, Crosby K, Seligson D, Palotie A, Inge L, Smith BL, Sawyers CL and Mischel PS: Analysis of the phosphatidylinositol 3'-kinase signaling pathway in glioblastoma patients in vivo. Cancer Res 63: 2742-2746, 2003.

3. Sumazin P, Yang X, Chiu HS, Chung WJ, Iyer A, Llobet-Navas D, Rajbhandari P, Bansal M, Guarnieri P, Silva J and Califano A: An extensive microRNA-mediated network of RNA-RNA interactions regulates established oncogenic pathways in glioblastoma. Cell 147: 370-381, 2011

4. Davis ME: Glioblastoma: Overview of disease and treatment. Clin J Oncol Nurs 20: S2-S8, 2016.

5. Schwartzentruber J, Korshunov A, Liu XY, Jones DT, Pfaff E, Jacob K, Sturm D, Fontebasso AM, Quang DA, Tonjes M, et al: Driver mutations in histone $\mathrm{H} 3.3$ and chromatin remodelling genes in paediatric glioblastoma. Nature 482: 226-231, 2012.

6. Frattini V, Trifonov V, Chan JM, Castano A, Lia M, Abate F, Keir ST, Ji AX, Zoppoli P, Niola F, et al: The integrated landscape of driver genomic alterations in glioblastoma. Nat Genet 45: 1141-1149, 2013.

7. Zhang Y, Li X, Zhou D, Zhi H, Wang P, Gao Y, Guo M, Yue M, Wang Y, Shen W, et al: Inferences of individual drug responses across diverse cancer types using a novel competing endogenous RNA network. Mol Oncol 12: 1429-1446, 2018.

8. Yu F, Quan F, Xu J, Zhang Y, Xie Y, Zhang J, Lan Y, Yuan H, Zhang H, Cheng S, et al: Breast cancer prognosis signature: Linking risk stratification to disease subtypes. Brief Bioinform: Sep 3, 2018 (Epub ahead of print).

9. Hayes J, Peruzzi PP and Lawler S: MicroRNAs in cancer: Biomarkers, functions and therapy. Trends Mol Med 20: 460-469, 2014.

10. Best MG, Sol N, Kooi I, Tannous J, Westerman BA, Rustenburg F, Schellen P, Verschueren H, Post E, Koster J, et al: RNA-Seq of tumor-educated platelets enables blood-based pan-cancer, multiclass, and molecular pathway cancer diagnostics. Cancer Cell 28: 666-676, 2015

11. Lai NS, Wu DG, Fang XG, Lin YC, Chen SS, Li ZB and Xu SS: Serum microRNA-210 as a potential noninvasive biomarker for the diagnosis and prognosis of glioma. $\mathrm{Br} \mathrm{J}$ Cancer 112: 1241-1246, 2015.

12. Gilder AS, Natali L, Van Dyk DM, Zalfa C, Banki MA, Pizzo DP, Wang H, Klemke RL, Mantuano E and Gonias SL: The urokinase receptor induces a mesenchymal gene expression signature in glioblastoma cells and promotes tumor cell survival in neurospheres. Sci Rep 8: 2982, 2018.

13. Zhang Y, Liu D, Wang L, Wang S, Yu X, Dai E, Liu X, Luo S and Jiang W: Integrated systems approach identifies risk regulatory pathways and key regulators in coronary artery disease. J Mol Med (Berl) 93: 1381-1390, 2015.

14. Jiang W, Zhang Y, Meng F, Lian B, Chen X, Yu X, Dai E, Wang S, Liu X, Li X, et al: Identification of active transcription factor and miRNA regulatory pathways in Alzheimer's disease. Bioinformatics 29: 2596-2602, 2013.

15. Stelzer G, Rosen N, Plaschkes I, Zimmerman S, Twik M, Fishilevich S, Stein TI, Nudel R, Lieder I, Mazor Y, et al: The genecards suite: From gene data mining to disease genome sequence analyses. Curr Protoc Bioinformatics 54: 1.30.1-1.30.33, 2016.

16. Jiang Q, Wang Y, Hao Y, Juan L, Teng M, Zhang X, Li M, Wang G and Liu Y: miR2Disease: A manually curated database for microRNA deregulation in human disease. Nucleic Acids Res 37 (Database Issu): D98-D104, 2009.

17. Durinck S, Spellman PT, Birney E and Huber W: Mapping identifiers for the integration of genomic datasets with the R/Bioconductor package biomaRt. Nat Protoc 4: 1184-1191, 2009.

18. Hsu SD, Lin FM, Wu WY, Liang C, Huang WC, Chan WL, Tsai WT, Chen GZ, Lee CJ, Chiu CM, et al: miRTarBase: A database curates experimentally validated microRNA-target interactions. Nucleic Acids Res 39 (Database Issue): D163-D169, 2011.

19. Wingender E, Chen X, Hehl R, Karas H, Liebich I, Matys V, Meinhardt T, Pruss M, Reuter I and Schacherer F: TRANSFAC: An integrated system for gene expression regulation. Nucleic Acids Res 28: 316-319, 2000 . 
20. Xiao F, Zuo Z, Cai G, Kang S, Gao X and Li T: miRecords: An integrated resource for microRNA-target interactions. Nucleic Acids Res 37 (Database Issue): D105-D110, 2009.

21. Sethupathy P, Corda B and Hatzigeorgiou AG: TarBase: A comprehensive database of experimentally supported animal microRNA targets. RNA 12: 192-197, 2006.

22. Zhao J, Yang TH, Huang Y and Holme P: Ranking candidate disease genes from gene expression and protein interaction: A Katz-centrality based approach. PLoS One 6: e24306, 2011.

23. R Core Team: R: A language and environment for statistical computing. R Foundation for Statistical Computing, Vienna, 2012. http://www.R-project.org/.

24. Yu G, Wang LG, Han Y and He QY: clusterProfiler: An R package for comparing biological themes among gene clusters. OMICS 16: 284-287, 2012.

25. The Gene Ontology Consortium: The gene ontology resource: 20 years and still GOing strong. Nucleic Acids Res 47: D330-D338, 2019.

26. Girvan M and Newman ME: Community structure in social and biological networks. Proc Natl Acad Sci USA 99: 7821-7826, 2002.

27. Osuka S and Van Meir EG: Cancer therapy: Neutrophils traffic in cancer nanodrugs. Nat Nanotechnol 12: 616-618, 2017.

28. Junttila MR and de Sauvage FJ: Influence of tumour micro-environment heterogeneity on therapeutic response. Nature 501: 346-354, 2013

29. Chakravarty D, Pedraza AM, Cotari J, Liu AH, Punko D, Kokroo A, Huse JT, Altan-Bonnet G and Brennan CW: EGFR and PDGFRA co-expression and heterodimerization in glioblastoma tumor sphere lines. Sci Rep 7: 9043, 2017.

30. Wang Y, Vogel G, Yu Z and Richard S: The QKI-5 and QKI-6 RNA binding proteins regulate the expression of microRNA 7 in glial cells. Mol Cell Biol 33: 1233-1243, 2013.

31. Wang B, Sun F, Dong N, Sun Z, Diao Y, Zheng C, Sun J, Yang Y and Jiang D: MicroRNA-7 directly targets insulin-like growth factor 1 receptor to inhibit cellular growth and glucose metabolism in gliomas. Diagn Pathol 9: 211, 2014.

32. Patel VN, Gokulrangan G, Chowdhury SA, Chen Y, Sloan AE, Koyuturk M, Barnholtz-Sloan J and Chance MR: Network signatures of survival in glioblastoma multiforme. PLoS Comput Biol 9: e1003237, 2013

33. Jhanwar-Uniyal M, Amin AG, Cooper JB, Das K, Schmidt MH and Murali R: Discrete signaling mechanisms of mTORC1 and mTORC2: Connected yet apart in cellular and molecular aspects. Adv Biol Regul 64: 39-48, 2017.
34. Beguerisse-Díaz M, Desikan R and Barahona M: Linear models of activation cascades: Analytical solutions and coarse-graining of delayed signal transduction. J R Soc Interface 13: pii: 20160409, 2016.

35. Pekarsky Y and Croce CM: Noncoding RNA genes in cancer pathogenesis. Adv Biol Regul 71: 219-223, 2019.

36. Yu F, Zhang G, Shi A, Hu J, Li F, Zhang X, Zhang Y, Huang J, Xiao Y, Li X and Cheng S: LnChrom: A resource of experimentally validated IncRNA-chromatin interactions in human and mouse. Database (Oxford) 2018, 2018.

37. Shao H, Chung J, Balaj L, Charest A, Bigner DD, Carter BS, Hochberg FH, Breakefield XO, Weissleder R and Lee H: Protein typing of circulating microvesicles allows real-time monitoring of glioblastoma therapy. Nat Med 18: 1835-1840, 2012.

38. Mischel PS, Shai R, Shi T, Horvath S, Lu KV, Choe G, Seligson D, Kremen TJ, Palotie A, Liau LM, et al: Identification of molecular subtypes of glioblastoma by gene expression profiling. Oncogene 22: 2361-2373, 2003.

39. Wheeler DL, Dunn EF and Harari PM: Understanding resistance to EGFR inhibitors-impact on future treatment strategies. Nat Rev Clin Oncol 7: 493-507, 2010.

40. Choi C, Xu X, Oh JW, Lee SJ, Gillespie GY, Park H, Jo H and Benveniste EN: Fas-induced expression of chemokines in human glioma cells: Involvement of extracellular signal-regulated kinase $1 / 2$ and p38 mitogen-activated protein kinase. Cancer Res 61: 3084-3091, 2001.

41. Pan YB, Zhang CH, Wang SQ, Ai PH, Chen K, Zhu L, Sun ZL and Feng DF: Transforming growth factor beta induced (TGFBI) is a potential signature gene for mesenchymal subtype high-grade glioma. J Neurooncol 137: 395-407, 2018.

42. Cordell HJ: Detecting gene-gene interactions that underlie human diseases. Nat Rev Genet 10: 392-404, 2009.

43. Cole BS, Hall MA, Urbanowicz RJ, Gilbert-Diamond D and Moore JH: Analysis of gene-gene interactions. Curr Protoc Hum Genet 95: 1.14.1-1.14.10,2017. 